\title{
The First Station of the Long Wavelength Array
}

\author{
Patricia Henning* \\ University of New Mexico \\ E-mail: henning@cosmos.phys.unm.edu
}

\section{Steven W. Ellingson}

Virginia Polytechnic Institute and State University

E-mail: ellingson@vt.edu

\author{
Gregory B. Taylor, Joseph Craig, Ylva Pihlström, Lee J Rickard \\ University of New Mexico \\ E-mail: gbtaylor@unm.edu, joecraig@unm.edu, ylva@unm.edu, \\ lrickard@unm.edu
}

\section{Tracy E. Clarke, Namir E. Kassim}

U.S. Naval Research Laboratory

E-mail: tracy.clarke@nrl.navy.mil, Namir.Kassim@nrl.navy.mil

\section{Aaron Cohen}

U.S. Naval Research Laboratory and Johns Hopkins Applied Physics Laboratory

E-mail: acohen00@gmail.com

The Long Wavelength Array (LWA) will be a new multi-purpose radio telescope operating in the frequency range 10-88 MHz. Upon completion, LWA will consist of 53 phased array "stations" distributed over a region about $400 \mathrm{~km}$ in diameter in the state of New Mexico. Each station will consist of 256 pairs of dipole-type antennas whose signals are formed into beams, with outputs transported to a central location for high-resolution aperture synthesis imaging. The resulting image sensitivity is estimated to be a few mJy $(5 \sigma, 8 \mathrm{MHz}, 2$ polarizations, $1 \mathrm{hr}$, zenith) in 20-80 MHz; with resolution and feld of view of $\left(8^{\prime \prime}, 8^{\circ}\right)$ and $\left(2^{\prime \prime}, 2^{\circ}\right)$ at $20 \mathrm{MHz}$ and $80 \mathrm{MHz}$, respectively. All 256 dipole antennas are in place for the frst station of the LWA (called LWA1), and commissioning activities are well underway. The station is located near the core of the EVLA, and is expected to be fully operational in early 2011.

ISKAF2010 Science Meeting

June 10 -14 2010

Assen, the Netherlands

\footnotetext{
* Speaker.
} 


\section{The Long Wavelength Array}

We are living in an era of resurgent interest in astronomy at long wavelengths, as witnessed by the development of new low frequency telescopes around the world, including LOFAR, MWA, PAPER, all discussed in this volume, and the LWA.

The LWA is designed for both long-wavelength astrophysics and ionospheric science. Science to be addressed by the LWA includes cosmic evolution, the acceleration of relativistic particles, physics of the interstellar and intergalactic media, solar science and space weather, and "discovery science"; that is, the search for previously unknown sources and phenomena [1]. Specif c objectives for LWA are spelled out in [3].

Upon completion, LWA will consist of 53 electronically-steered phased array "stations," each consisting of 256 pairs of dipole-like antennas, operating with Galactic noise-limited sensitivity over the frequency range $20-80 \mathrm{MHz}$, with reduced sensitivity over $10-88 \mathrm{MHz}$. The stations will be distributed over the state of New Mexico, (Figure 1), with maximum baselines of up to $400 \mathrm{~km}$, yielding resolution of $8^{\prime \prime}$ and $2^{\prime \prime}$ at $20 \mathrm{MHz}$ and $80 \mathrm{MHz}$ respectively. Beams formed by the stations will be transmitted to a central location and correlated to form images using aperture synthesis techniques. The full array is expected to reach mJy-class sensitivity. Stations will also be capable of operating as independent radio telescopes.

\section{LWA-1, the First Station of the LWA}

The f rst station of the LWA, called LWA-1, is being constructed near the core of the EVLA. Its 256 crossed dipole antennas (shown in Figure 2) were completed in November 2009. The elements are distributed in a $\sim 100$-m aperture, producing beam FOV of 8 deg and 2 deg at $20 \mathrm{MHz}$ and $80 \mathrm{MHz}$. Every element is digitized to allow independent pointings of beams, and all-sky snapshot imaging. Using 256 dual-polarization antennas results of spacings of $3 \times$ Nyquist at 80 MHz. The pseudorandom distribution of the antennas mitigates against aliasing (Figure 3). The minimum separation between antennas of $5 \mathrm{~m}$ allows easy access for maintenance, and also reduces beam desensitization due to sky noise correlation [4]. Various ionospheric, solar, and especially Galactic science goals require the ability to observe towards declinations which appear low in the southern sky from New Mexico. To compensate for the elevation-plane widening of the beam for these observations, the station footprint has been made somewhat elliptical with a NS:EW axial ratio of 1.1 .

\subsection{RF Signal Path, and System Status}

The signal from every antenna is processed by a dedicated direct-sampling receiver consisting of an analog receiver (ARX) and an analog-to-digital converter (A/D) which samples 196 million samples per second (MSPS). Beams are formed using a time-domain delay-and-sum architecture, which allows the entire 10-88 MHz passband associated with each antenna to be processed as single wideband data stream. Eventually beams from the stations will be correlated in a central location, but for individual stations it is also possible to record the beam data for later processing. To facilitate commissioning activities, diagnostics, and certain types of science observations requiring all-sky f eld-of-view (FOV), the station electronics will also have the capability to coherently 


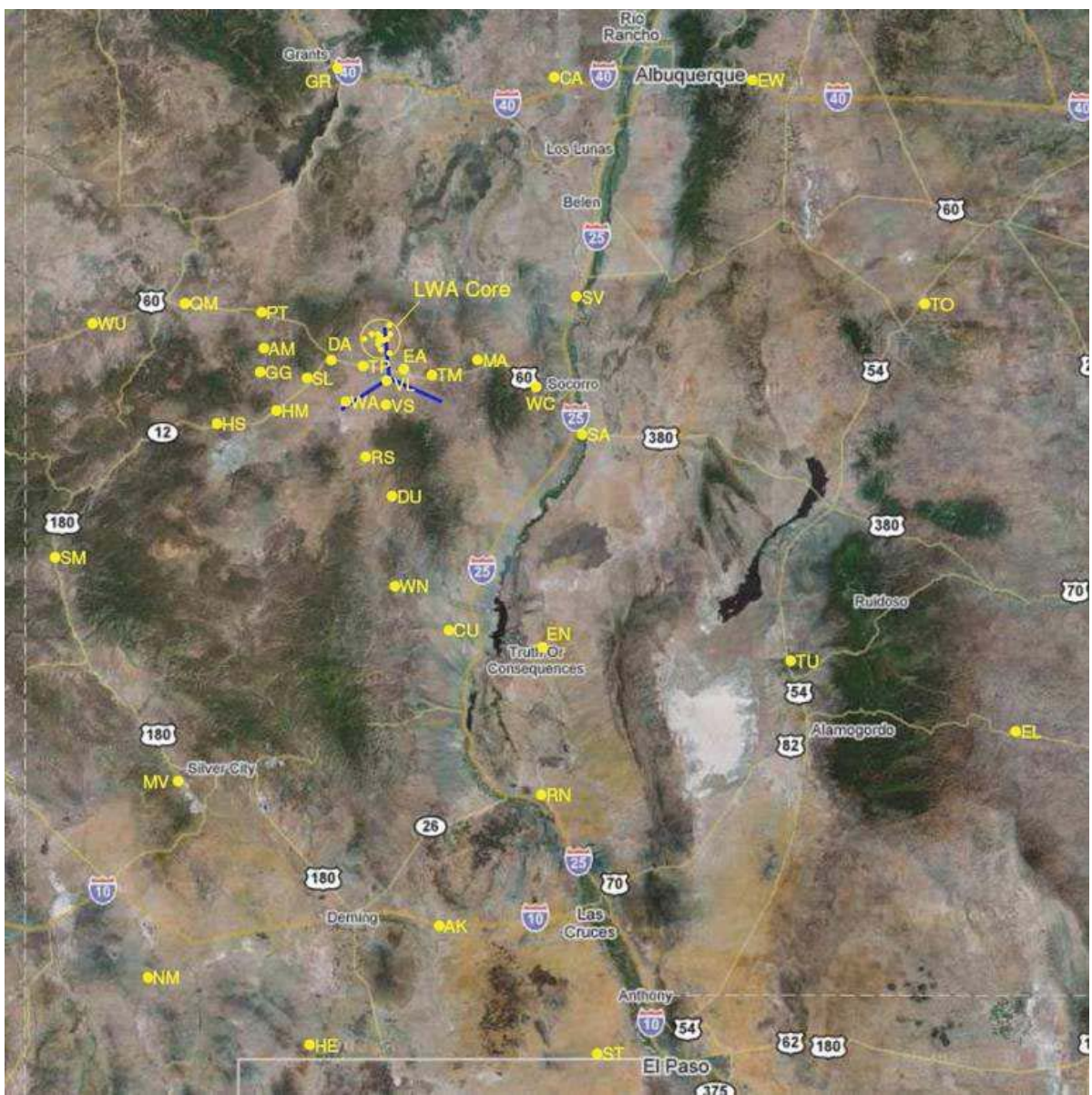

Figure 1: Planned LWA station locations across the state of New Mexico. The "Y" of the EVLA is shown in blue.

capture and record the output of all $\mathrm{A} / \mathrm{Ds}$, where each $\mathrm{A} / \mathrm{D}$ corresponds to one antenna. This will occur in two modes: the "transient buffer - wideband" (TBW) allows the raw output of the A/Ds to be collected continuously, but only for $\sim 100 \mathrm{~ms}$ at a time. The "transient buffer - narrowband" (TBN), in contrast, allows a single tuning of $\sim 100 \mathrm{kHz}$ bandwidth to be recorded indef nitely.

To accommodate the various uncertainties in the RFI environment, we have developed an ARX which can be electronically reconf gured between three modes: A full-bandwidth (10-88 MHz) uniform-gain mode, a full-bandwidth dual-gain mode in which frequencies below about $40 \mathrm{MHz}$ can be attenuated using a "shelf flter," and a 28-54 MHz mode, which serves as a last line of defense in the case where RFI above and/or below this range is persistently linearity-limiting. A sample spectrum, showing the strong RFI at the top and bottom ends of the spectrum, is shown in Figure 4. The noise is dominated by that from the sky, as can be seen by comparison of the sky signal with that of a terminated load.

The installation of the dipole antennas for LWA-1 is complete. At the time of writing (July 2010), cabling is on track for completion in August 2010. The system is currently operating with an interim 16-antenna analog beamformer, using the Eight-meter-wavelength Transient Array (ETA) 


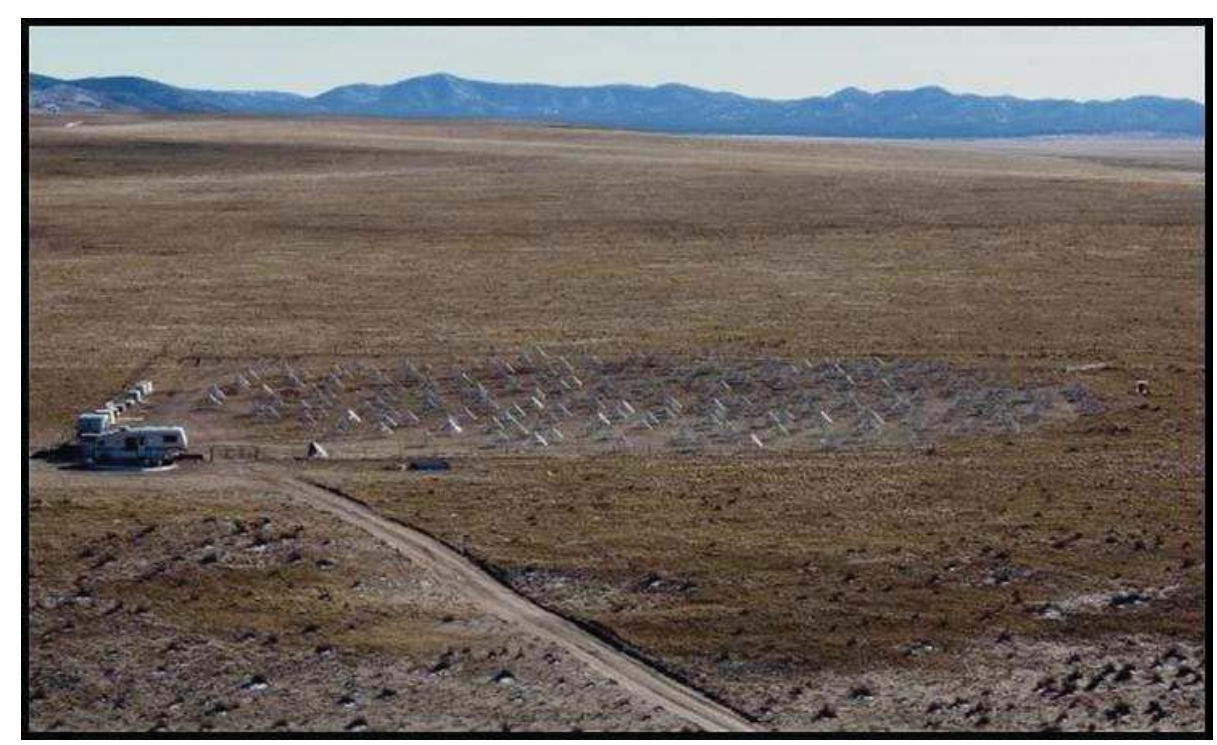

Figure 2: The frst station of the LWA, as seen from the EVLA antenna assembly building. The equipment shelter is seen to the left.

"S60" digital receiver, and data capture system. In Figure 5 we plot the total power received for a single dipole and for 7 dipoles summed together over the course of three days. Some discrepant values are the result of thunderstorm activity on the afternoon of April 15.

\subsection{LWA-1 Sensitivity}

At low frequencies, Galactic noise can be a signif cant or dominant contribution to the total noise. This, combined with mutual coupling between antennas, makes it diff cult to predict the sensitivity of these instruments. Ellingson (2010)[6] describes a system model and procedure for estimating the system equivalent fux density (SEFD) - a useful and meaningful metric of the sensitivity of a radio telescope - that accounts for these issues. The method is applied to LWA-1, and it is shown that the correlation of Galactic noise between antennas signif cantly desensitizes the array for beam pointings that are not close to the zenith. It is also shown that considerable improvement is possible using beamforming coeff cients that are designed to optimize signal-tonoise ratio under these conditions (see Figure 6). The receiver noise is about $250 \mathrm{~K}$, but has little inf uence on the SEFDs which range between 3,000 and 100,000 Jy over the frequency and elevation range plotted.

\subsection{Radio Frequency Interference and Deep Integrations}

Radio Frequency Interference (RFI) is always an issue when working at low frequencies. We have sought to minimize internally-generated RFI by use of careful shielding of the station electronics. For externally-generated RFI we have chosen modes and gain settings based on a detailed study of RFI at the EVLA, combined with a study of A/D capabilities, leading to the conclusion that an A/D of about 200 MSPS with 8-bit sampling was probably suff cient when combined with an ARX having the capabilities described above. We currently favor a sampling rate $F_{s}=196$ MSPS, 


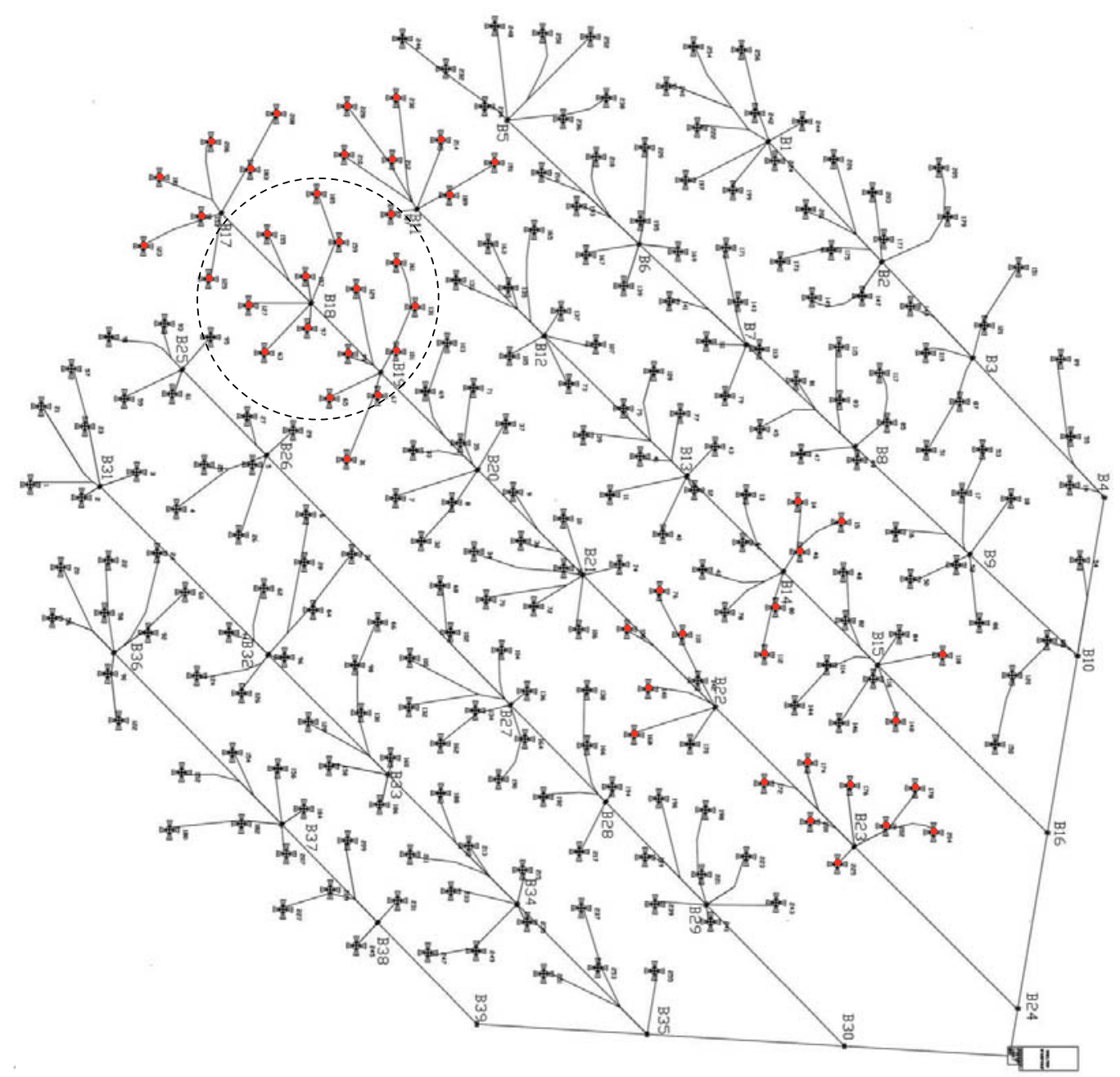

Figure 3: Diagram of antenna locations and cabling in LWA-1. The circle indicates antennas which participated in early tests.

as this results in the highly desirable situation that the $88-108 \mathrm{MHz}$ FM broadcast band aliases onto itself, which greatly reduces anti-alias $\mathrm{f}$ ltering requirements.

During construction of the frst station, the RFI situation has actually improved with the conversion from analog to digital television (Fig. 7). At the time of the conversion in July 2009, all of the television stations in New Mexico elected to relocate their digital transmissions to frequencies above the LWA band.

Strong RFI can cause a host of problems including compression in the receivers, and aliasing. Dealing with strong RFI requires the appropriate design choices, especially in the analog receiver, as described previously. Weak RFI can be just as damaging if it masks the faint cosmic signals that one is searching for. While station electronics are still being completed, deep integrations with a portion of the array show that the noise behaves radiometrically beyond an hour of data collecting (Fig. 8). These results were obtained at night, with $3 \mathrm{MHz}$ bandwidth, centered near $74 \mathrm{MHz}$. About $20 \%$ of the frequency band was excised due to the presence of low-level RFI in 


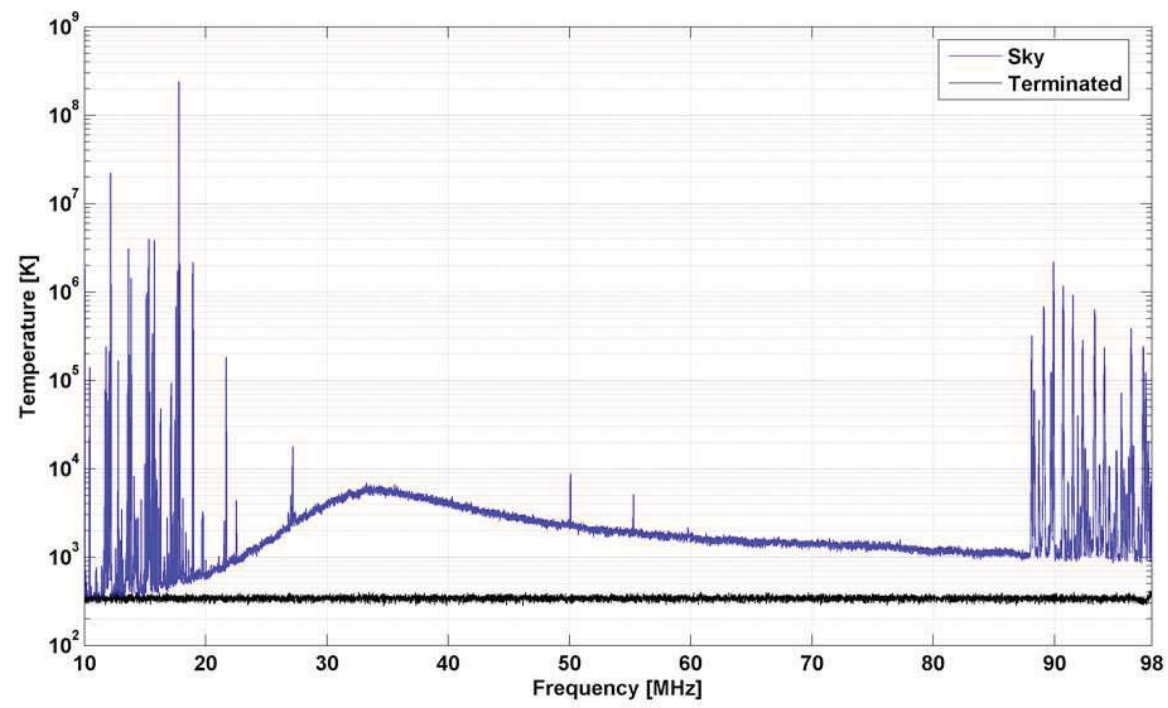

Figure 4: A spectrum from the summed 7-element beamformer after 10 seconds of integration with a spectral resolution of $6 \mathrm{kHz}$. The receiver noise temperature is about $250 \mathrm{~K}$ and roughly constant across the LWA frequency range (black line). The signal from the sky is shown in blue. The turnover in the sky signal near $33 \mathrm{MHz}$ is a result of the decreasing eff ciency of the LWA dipoles.

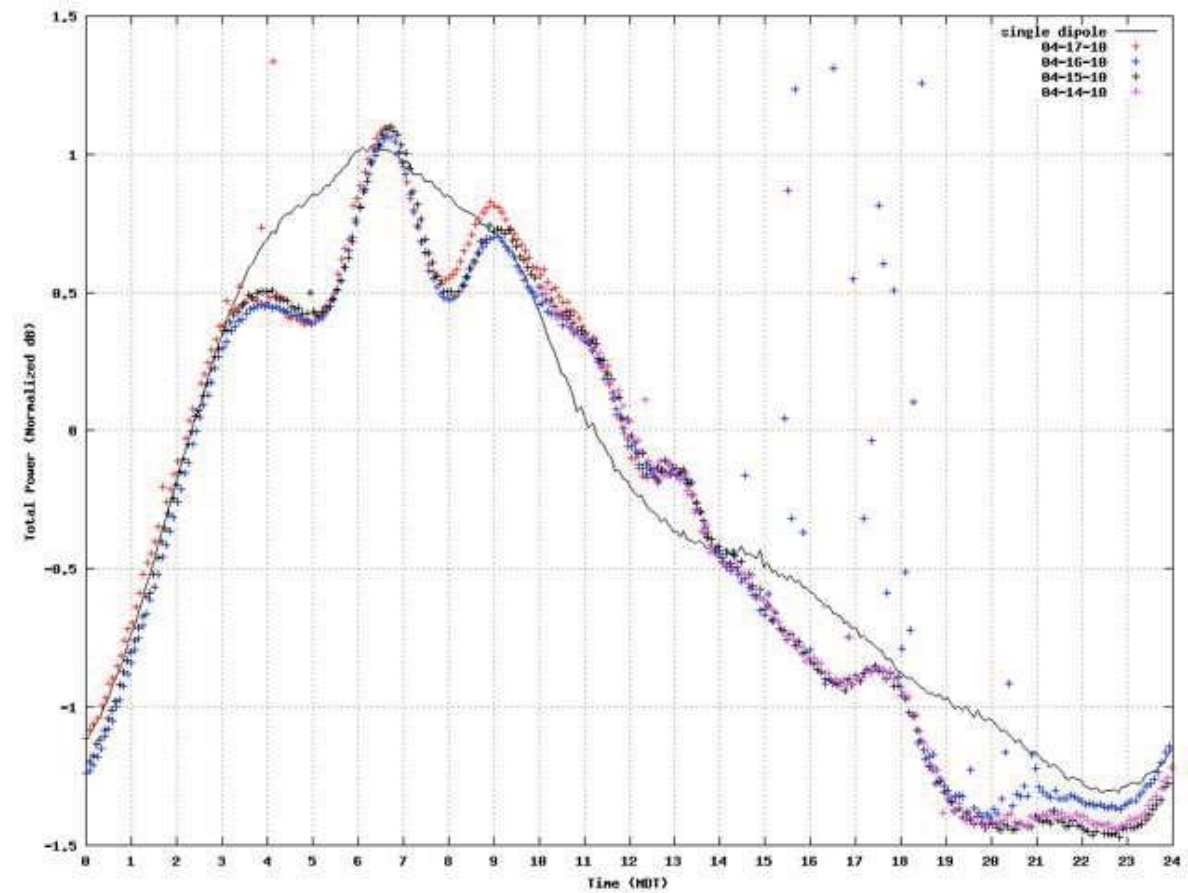

Figure 5: Plot showing total power for a single dipole (thin black line), integrating for 2 seconds every 5 minutes and for the sum of 7 dipoles (crosses) integrating in a similar fashion on three consecutive days. The discrepant high points are most likely due to thunderstorm activity. The frequency employed was 72.25 $\mathrm{MHz}$ with a $3 \mathrm{MHz}$ bandwidth. 


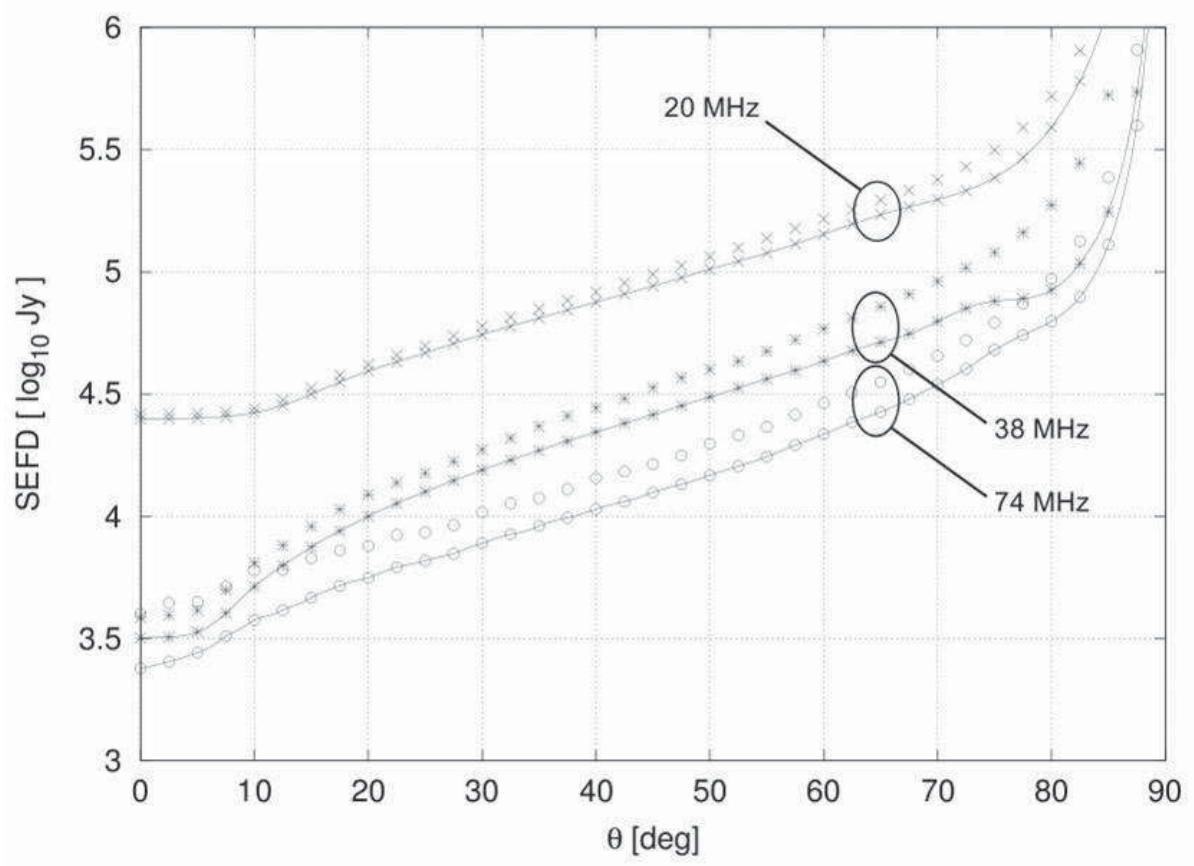

Figure 6: Calculated SEFD of LWA-1 as a function of degrees away from the zenith. For each frequency, the upper (dotted) curve is the result for simple beamforming, and the lower (solid) curve is the result for optimal beamforming. From [6].

this test. Deep integrations have paused while the array is being f nished, so the duration of the deepest integrations may well be longer that the hour+ limit indicated by current tests. This is indeed promising for the prospects of deep imaging.

\subsection{Digital Signal Processing}

The signal from every antenna is processed by a dedicated direct-sampling receiver consisting of an analog receiver (ARX) and an analog-to-digital converter (A/D) as described in $§ 2.1$. Beams are formed using a time-domain delay-and-sum architecture, which allows the entire $10-88 \mathrm{MHz}$ passband associated with each antenna to be processed as single wideband data stream. Delays are implemented in two stages: A coarse delay is implemented using a frst-in f rst-out (FIFO) buffer operating on the A/D output samples, followed by a f nite impulse response (FIR) flter, which is also used to introduce corrections for polarization and other frequency-dependent effects. The raw linear polarizations are transformed into calibrated standard orthogonal circular polarizations, and The signals are then added to the signals from other antennas processed similarly. Four dualpolarization beams of bandwidth $78 \mathrm{MHz}$, each capable of fully-independent pointing over the visible sky, will be constructed in this fashion.

The beams will be available for various "backends" implemented at the station level, such as data recorders, wideband spectrometers, and pulsar machines. For interferometric imaging, two "tunings" will be extracted from any frequency in the $78 \mathrm{MHz}$-wide passband, having bandwidth 

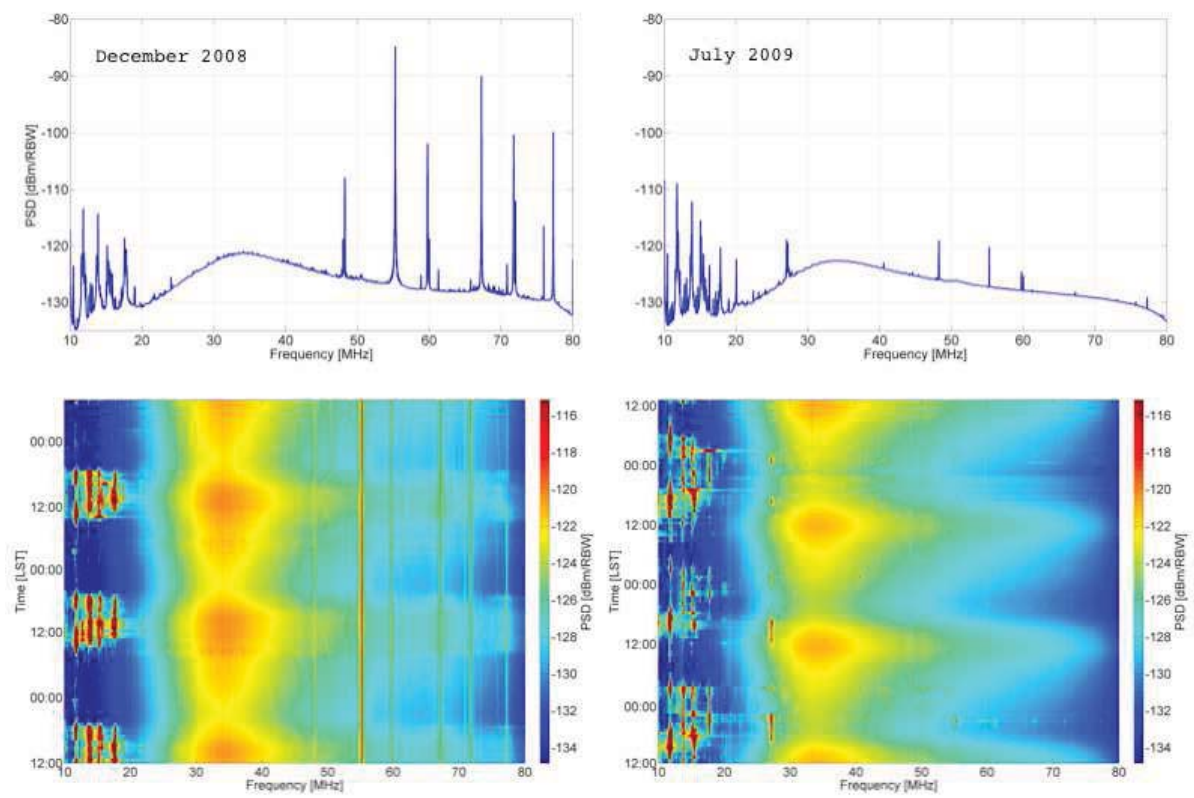

Figure 7: RFI environment at LWA-1. The left panels show an indicative spectrum and waterfall plot before the switch to digital TV. The right panels show data taken after the strong analog TV stations have vacated the spectrum. The spectrum is Galactic noise dominated. The diurnal variations seen in the waterfall plots are predominantly due to the culmination of the Galactic Center, and day-night variation of HF noise.

selectable between $400 \mathrm{kHz}$ and $20 \mathrm{MHz}$ divided into 4096 spectral channels. This is the output to the LWA correlator.

\subsection{Prototype All Sky Imager}

The Prototype All Sky Imager (PASI) will consist of a software correlator, and a near real time imager, both operating on an IBM cluster already purchased for these tasks. The PASI will be a "back-end" instrument designed specif cally for the f rst station of the LWA. Together, this equipment will allow us to image $75 \%$ of the sky every 24 hours, with an instantaneous feldof-view of over $120^{\circ} \times 120^{\circ}$. This will provide unparalleled resolution and sensitivity all-sky imaging in a largely unexplored frequency band. Expected sources to be imaged include the Sun, planets, f aring stars, active galaxies, quasars, magnetars, black holes, and gamma-ray bursts. The discovery of entirely new classes of objects are possible and will be followed up by observations with the LWA and other ground (e.g., EVLA) and space-based (e.g., Chandra) facilities.

\section{Timeline and Future Plans}

Currently the frst LWA station is using analog beamformers and the ETA "S60" digital receiver to commission the station. During summer 2010 this system will be increased to make use of up to 32 antennas. At the same time, the frst digital processing board will be installed with the 

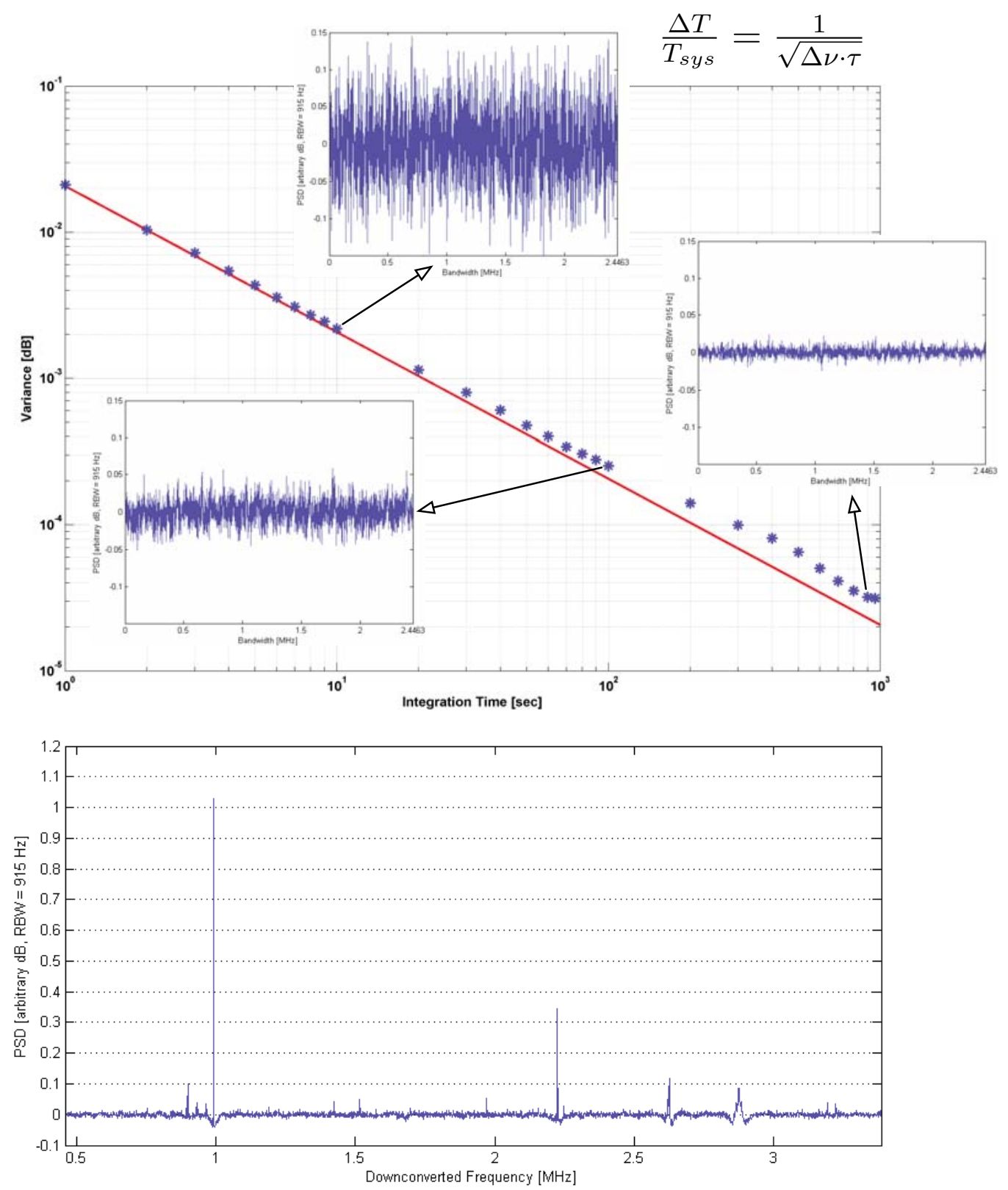

Figure 8: Deep integration noise behavior. The noise continues to drop as $1 / \sqrt{t}$ for integrations as long as an hour. These drift scan data were taken at night, with $2.45 \mathrm{MHz}$ bandpass (lower panel), centered at 72.25 MHz. About 2600 channels are shown. Data were corrected for diurnal total power variation. (The step at about $100 \mathrm{sec}$ is possibly due to HVAC turning on.) Total integration times where noise continues to drop radiometrically have exceeded an hour, beyond the time indicated here. 


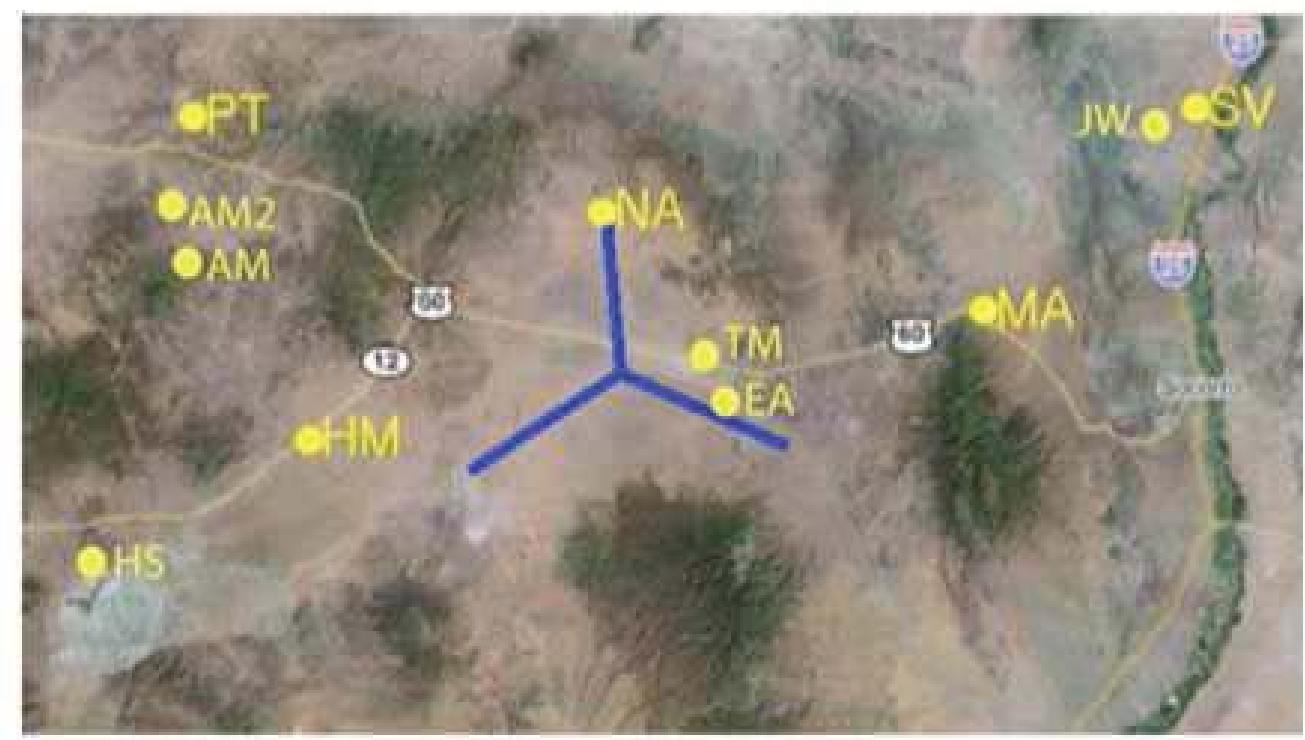

Figure 9: Plot showing planned stations, including LWA-2 (North Arm) and LWA-3 (Horse Mountain).

capability of handling up to 20 antennas. This will allow for commissioning of the transient buffer modes (TBW and TBN) initially, and later the beam outputs. The installation of the complete ana$\log$ and digital electronics will continue throughout the fall resulting in a fully operational station in early 2011. The f rst observing programs have already been selected and some of them have even begun taking data.

Beyond the f rst LWA station we are actively seeking funding for additional stations. The land and infrastructure for a second LWA station (labeled NA in Figure 9) is in place, 19 km north of LWA1. The land for a third station at Horse Mountain (HM) is also leased, but has not yet been developed. Leases for two other sites (marked MA and HS in Figure 9) are pending.

Basic research in radio astronomy at the Naval Research Laboratory is supported by 6.1 base funding.

\section{References}

[1] N.E. Kassim et al., "The Long Wavelength Array,” in [2], pp. 392-398.

[2] N. E. Kassim, M. R. Perez, W. Junor, and P. A. Henning (eds.), Clark Lake to the Long Wavelength Array: Bill Erickson's Radio Science, ASP Conf. Ser., Volume 345, 2005.

[3] T.E. Clarke, "Scientif c Requirements for the Long Wavelength Array," Ver. 2.3, Memo 117 in [5], Nov 19, 2007.

[4] Ellingson, S.W., Clarke, T.E., Cohen, A., Craig, J., Kassim, N.E., Pihlström, Y.M., Rickard, L.J., \& Taylor, G.B.,"The Long Wavelength Array”, Proc IEEE, 97, 1421, 2009.

[5] Long Wavelength Array Memo Series, [on-line] http://www.phys.unm.edu/ lwa/memos.

[6] S.W. Ellingson, "Sensitivity of Antenna Arrays for Long-Wavelength Radio Astronomy," IEEE, Trans. Ant. \& Prop., in press [Memo 166 in [5]] 